Original article

\title{
Epidemiologic survey of extra-pulmonary tuberculosis in Ahvaz from 2008 to 2013
}

\author{
Fatemeh Ahmadi ${ }^{\mathrm{a}}$, Mohammad Javad Mohammadi ${ }^{\mathrm{b}}$, Ammar Helalinasab $^{\mathrm{c}}$, \\ Shokrollah Salmanzadeh ${ }^{\mathrm{a}, * *}$ \\ a Infectious and Tropical Diseases Research Center, Health Research Institute, Ahvaz Jundishapur University of Medical Sciences, Ahvaz, Iran \\ ${ }^{\mathrm{b}}$ Department of Environmental Health Engineering, School of Public Health AND Air Pollution and Respiratory Diseases Research Center, Ahvaz Jundishapur University of \\ Medical Sciences, Ahvaz, Iran \\ ${ }^{\mathrm{c}}$ Ahvaz Jundishapur University of Medical Sciences, Ahvaz, Iran
}

\section{A R T I C L E I N F O}

\section{Keywords:}

Epidemiologic

Tuberculosis

Extra-pulmonary

Iran

\begin{abstract}
A B S T R A C T
Introduction: Tuberculosis (TB) still remains a major problem in public health. Recent researches have shown that the prevalence of Extra-pulmonary TB (EPTB) may vary according to geographic area and population. EPTB forms present with a variety of clinical characteristics. EPTB may be associated with a pulmonary localisation, which should be searched for whenever EPTB is diagnosed or suspected.

Objective: The aim of this study is to review the epidemiological feature of EPTB in Ahvaz, southwest of Iran. Materials and methods: In this retrospective study, medical records of all notified TB cases in TB data registry in Ahvaz Health Center over the 5-year period (2008-2013) were reviewed. Variables such as age, gender, location (urban or rural), the organs involved, the date of onset of symptoms and treatment, concomitant lung involvement, the presence of risk factors and side effect of treatment, if any, were considered.

Results: From a total of 1654 cases of TB, 471 cases (28\%) were EPTB; of them 201 patients (47/3\%) were male and $270(57 / 3 \%)$ were female. The commonest sites of involvement respectively were lymph nodes (32.3\%), pleura (27.2\%), bone and joint (8.1\%), gastrointestinal (5.7\%), genitourinary system (3.6\%).

Conclusion: This study demonstrates that TB still present as a health problem in Ahvaz. About one third of the TB cases were EPTB. It was more common in females and in 15-40 years old cases. The common sites of involvement were lymph nodes, pleura and bone and/or joints.
\end{abstract}

\section{Introduction}

Tuberculosis (TB) still remains a major problem in public health. It is second only to human immunodeficiency virus (HIV) as a cause of death worldwide resulting from a single infectious agent. TB can affect virtually every organ, most importantly the lungs, and is typically associated with granuloma formation. M.tuberculosis infects one third of the world's population and causes 8.7 million new cases of tuberculosis and approximately 1.4 million deaths each year. ${ }^{1}$ Before knowing the HIV infection $80 \%$ of TB cases were confined to lung. To two-thirds of patients with HIV co-infection extra-pulmonary TB (EPTB) is seen either alone or with lung involvement. ${ }^{2,3}$ Based on result several studies, the prevalence of EPTB may vary according to geographic area and population. HIV infection, Asian and African origin and Young age are independent risk factors for EPTB. Some studies have shown the proportion of EPTB is increasing which can be due to the spread of AIDS and greater access to diagnostic facilities. EPTB diagnosis is often difficult and requires invasive procedures such as biopsy. Delay in diagnosing the symptoms and the treatment of PTB and EPTB is a major cause of the disease spread and side effects. ${ }^{4}$

Obviously, awareness about prevalence of PTB and demographic and epidemiologic characteristics of the disease demonstrates the national control program accuracy in way of diagnosing TB cases and in other hand leads identifying high-risk groups and risk factors and so can cause in early detection of the disease and reduce the burden of symptoms and morbidity in the community.

The aim of this study is to review the epidemiological feature of EPTB in Ahvaz, southwest of Iran.

\footnotetext{
* Corresponding author. Infectious and Tropical Diseases Research Center, Health Research Institute, Ahvaz Jundishapur University of Medical Sciences, Ahvaz, Iran, Tel.: + 989166164837 .

E-mail address: salmanidmd@yahoo.com (S. Salmanzadeh).
} 


\section{Materials and Methods}

In a retrospective study, medical records of all notified TB cases in TB data registry in Ahvaz Health Centers over the 5-year period (2008-2013) were reviewed. All the EPTB cases were entered the study and cases with incomplete data were excluded. Identification of TB include pathological studies on samples obtained from biopsy, sputum culture and urine, spinal fluid examination and any necessary diagnostic imaging studies were included. Variables such as age, gender, location (urban or rural), the organs involved, the date of onset of symptoms and treatment, concomitant lung involvement, the presence of risk factors and side effect of treatment, if any, were considered. Analysis results using descriptive statistics such as mean and percentile calculating was performed using SPSS software, ver16.

\section{Results}

From 2008 until 2013 a total of 1654 cases of TB in health center of Ahvaz were diagnosed which 471 cases (28\%) of them were ЕРTB. 4 cases had incomplete information, who were excited from the study. Of these, 201 patients $(47 / 3 \%)$ were male and $270(57 / 3 \%)$ were female. The mean age, over ally, was 37 years (Fig. 1).

The mean age of patients with EPTB according to gender was 36 years for females and 37 years for males (Table 1).

Among the 471 cases were recorded, 22 patients (4.7\%) had both pulmonary TB (РTB) and ЕРTB and the remaining 449 patients (95.3\%) had only EPTB. 438 of the patients (93.2\%) were living in urban areas and 32 patients $(6.8 \%)$ were living in rural. According to total populations in these two areas, the proportion calculated by Minitab showed significant differences between them, where in urban areas was higher. Among the patients just 6 (1.3\%) of them where immigrants and the rest where Iranians. Sites of EPTB according to gender illustrated in Fig. 2. The most common site of EPTB was the lymph nodes with 152 cases $(32.3 \%)$ followed by the pleura 128 cases $(27.2 \%)$, bone and joint involvement with 38 cases (8.1\%), gastrointestinal (GI) involvement with 27 cases $(5.7 \%)$ and genitourinary system with 17 cases (3.6\%). Other sites include, respectively, the nervous system, miliary tuberculosis, skin, pericardium and about 61 cases (13\%) had other sites
Table 1

Demographic and clinical characteristics.

\begin{tabular}{|c|c|}
\hline Characteristics & EPTB patients n (\%) \\
\hline \multicolumn{2}{|l|}{ Age } \\
\hline mean (male/female) & $37(37 / 36)$ \\
\hline$<15$ & $35(7.4 \%)$ \\
\hline $15-25$ & $87(18.5 \%)$ \\
\hline $25-40$ & $186(39.5 \%)$ \\
\hline $40-65$ & $132(28 \%)$ \\
\hline$>65$ & $31(6.6 \%)$ \\
\hline \multicolumn{2}{|l|}{ Gender } \\
\hline Male & $201(47.3 \%)$ \\
\hline female & $270(57.3 \%)$ \\
\hline \multicolumn{2}{|l|}{ TB } \\
\hline EPTB alone & $449(95.3 \%)$ \\
\hline both РТВ \& ЕРТВ & $22(4.7 \%)$ \\
\hline \multicolumn{2}{|l|}{ Living place } \\
\hline Urban & $438(93.2 \%)$ \\
\hline rural & $32(6.8 \%)$ \\
\hline \multicolumn{2}{|l|}{ Nationality } \\
\hline Iranian & $465(98.7 \%)$ \\
\hline Immigrant & $6(1.3 \%)$ \\
\hline \multicolumn{2}{|l|}{ Underlying disease } \\
\hline No disease & $427(90.9 \%)$ \\
\hline diabetes mellitus & $20(4.2 \%)$ \\
\hline Addiction & $8(1.7 \%)$ \\
\hline Silicosis & $8(1.7 \%)$ \\
\hline CKD & $2(0.4 \%)$ \\
\hline Cancer & $1(0.2 \%)$ \\
\hline silicosis + addiction & $4(0.8 \%)$ \\
\hline \multicolumn{2}{|l|}{ Side effects } \\
\hline No side effect & $482(98.1 \%)$ \\
\hline drug hepatitis & $5(1.1 \%)$ \\
\hline vomit \& blurred vision & $3(0.6 \%)$ \\
\hline skin rash & $1(0.2 \%)$ \\
\hline \multicolumn{2}{|l|}{ Prison } \\
\hline No & $455(96.6 \%)$ \\
\hline Yes & $16(3.4 \%)$ \\
\hline
\end{tabular}

involvement including breast, liver, kidneys, eyes, spleen, larynx and thyroid. Although lymph nodes involvement was the predominant site of ЕРТВ in women in men pleural involvement had the highest frequency. About the presence of underlying diseases and risk factors it
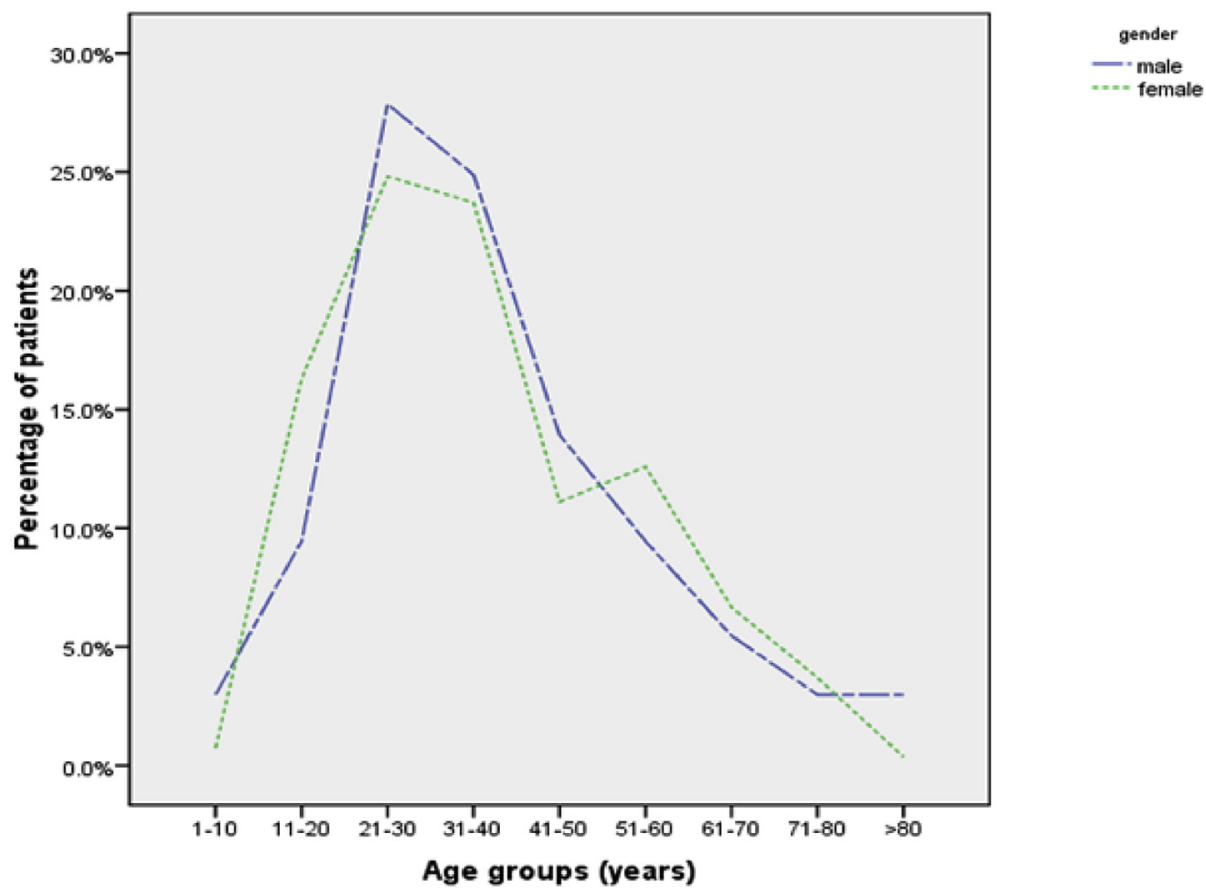

Fig. 1. Proportional age distribution for EPTB among the patients stratified by gender. 

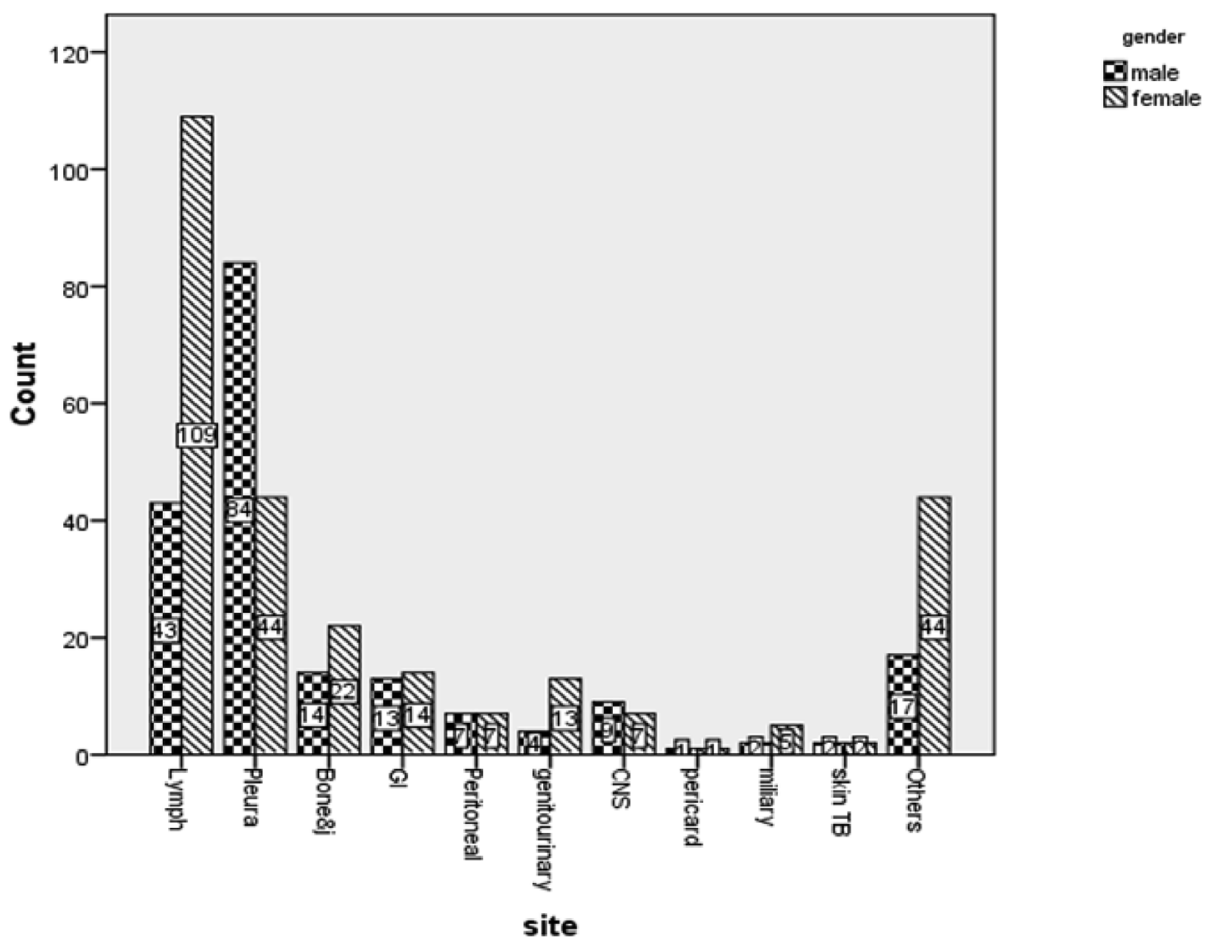

Fig. 2. Distribution of sites of extra-pulmonary tuberculosis.

was found that about 427 cases (90.9\%) had no underlying disease and 20 patients (4.2\%) had diabetes, $8(1.7 \%)$ had addiction, 8 had silicosis $(1.7 \%), 2(0.4 \%)$ had chronic kidney disease, 1 had cancer $(0.2 \%)$, and $4(0.8 \%)$ were recorded to had silicosis and opium addiction. Among the patients 16 cases (3.4\%) had a history of incarceration. The mean duration time from the clinical manifestation to starting the treatment was 178 days and the duration for the one-fourth was more than 249 days. In terms of side effects during anti-TB treatment the records showed that 5 patients (1.1\%) developed drug induced hepatitis, 1 case $(0.2 \%)$ showed skin rash and 3 cases $(0.6 \%)$ had blurred vision.

\section{Discussion}

In our study $28 \%$ of the patients (nearly one-third) were EPTB cases. Different studies showed a $12-29 \%$ proportions $^{5-7}$ which shows the differences in different geographical regions (6). In a previous study in Khuzestan province $836.7 \%$ of patients were EPTB cases. This difference can be explained because of the different study populations and perhaps can reveal a better diagnostic and treatment facilities in Ahvaz rather than the other cities in the province. In our study $57.3 \%$ of the cases were female, which is consistent with previous studies. ${ }^{3,5,6}$ In a large population-based case-control study characterizing EPTB in Arkansas's, being female was identified an independent risk factor for EPTB. ${ }^{7}$ Different explanation such as social, cultural and biological causes has been mentioned ${ }^{7,9}$ but obvious biological credibility remains to be determined through further investigations. Mean age in the patients was 37 years old. There wasn't significant differences in mean age between males and females. As is shown in Table 1 , we found that $58 \% \%$ of the patients are between 15 and 40 years old. Our finding was consistent with previous studies ${ }^{3,5,6,9}$ where EPTB was more common in young and middle age's patients. Other studies in USA and Turkey ${ }^{10}$ have reported that age was not associated with EPTB. These inconsistencies could be due to differences in prevalence of host-related factors or important co-exposures. ${ }^{10}$ In our study the proportion of the patients living in urban area was significantly higher. This finding is inconsistent with a study in Gorgan, north east of $\operatorname{Iran}^{6}$ and perhaps this can be explained by a better preventive care and more active family medicine project in rural area rather than urban in Iran.

As is shown in Fig. 1, most common sites of involvement were lymph nodes and pleura, respectively. Earlier studies have revealed that sites of involvement in ЕРTB may be variable. In a study in Gorgan ${ }^{6}$ the common site was pleura following by lymph nodes. In Turkey ${ }^{11}$ the genitourinary system and meningeal TB wer e the common sites, whereas in the USA, ${ }^{7}$ bones and/or joints were the most common sites. Our study is comparable to studies from Zahedan, south east of Iran ${ }^{5}$ which reported that lymph nodes were the commonest sites of EPTB. In the case of underlying diseases we couldn't gather complete data about concomitant HIV infection due to incomplete data registry in Ahvaz health center, especially in the earlier years of the study. But about the other diseases our finding showed that diabetes mellitus (DM) was the common underlying disease in the EPTB patients. However, in $90.9 \%$ of the patients not any underlying disease was notified. In a previous study in USA $^{12}$ the effect of comorbid diseases have been studied where positive HIV determined as a factor associated with EPTB and DM was associated with PTB and not EPTB. Our study didn't show a significant correlation between the underlying diseases and ЕРTB. However, the possibility of deficits in case registration of the Health Centers must be considered too.

In the study population mean duration time from the clinical manifestation to starting the treatment was 178 days and among the patients started the treatment just 9 patients (1.9\%) had drug reactions. In previous studies the mentioned variables were not studied. The duration time can be used as a factor showing people and health system's power for controlling diseases. The relatively low proportion of drug reactions shows a relatively good tolerance to antituberculosis drugs in the study population.

As has been shown that crowded living conditions can increase the risk of infection ${ }^{1}$ we studied the possible history of recent beings in jails and about 16 cases $(3.4 \%)$ had positive history. Perhaps a better factor to evaluate the crowding effect such as population concentration in living spaces is needed. 


\section{Conclusion}

This study demonstrates that TB still present as a health problem in Ahvaz. About one third of the TB cases were EPTB. It was more common in females and in 15-40 years old cases. The common sites of involvement were lymph nodes, pleura and bone and/or joints and a more precise data registering is needed to detect the burden of HIV coinfection in the city.

\section{Funding/Support}

This work was financially supported by grant: (TB-06) from Ahvaz Jundishapur University of Medical Sciences.

\section{Declaration of competing interest}

All authors have no conflict of interests.

\section{Acknowledgements}

This article was written from the thesis of Ammar Helalinasab with the same title. The authors would like to thank Ahvaz Jundishapur University of Medical Sciences, Iran for providing financial supported by the grant: (TB-06) of this research.

\section{REFFERENCES}

1. Bennett JE, Dolin R, Blaser MJ. Mandell, Douglas, and Bennett's Principles and Practice of Infectious Diseases. Elsevier Health Sciences; 2014.

2. Golden MP, Vikram HR. Extrapulmonary tuberculosis: an overview. Am Fam Physician. 2005;72:1761-1768.

3. SGa Mousavi, Saberi Hr, Sharif Ar, et al. A comparative study of patients with pulmonary tuberculosis and extra- pulmonary tuberculosis in Kashan. KAUMS J ( FEYZ ). 2009;13:235-241.

4. Shafigh E, Siadati S. Extra pleuro-pulmonary tuberculosis in Babol Shahid Beheshti hospital during 14 years. J Gorgan Univ Med Sci. 2004;6:61-65.

5. M M, M S, B Sm, AR J, Z R. Epidemiology of extra pulmonary tuberculosis in Zahedan Zahedan J Res Med. Sci.4:275 - 281

6. Khodabakhshi B, Jabari A, Besharat S. Epidemiology of extra pulmonary tuberculosis in Gorgan. Iranian J Infectious Dis Trop Med. 2008;40:43-46.

7. Yang Z, Kong Y, Wilson F, et al. Identification of risk factors for extrapulmonary tuberculosis. Clin Infect Dis. 2004;38:199-205.

8. Mohammad AS, Nejad S. The causes and risk factors of tuberculosis deaths in Khuzestan. Acta Med Iran. 2009;47:89-92.

9. Ibraheem SA, YrI Salih, Saad H. Extra pulmonary tuberculosis among patients attended the consultation clinic of respiratory diseases in Salahiddin Governorate; an epidemiological study. Tikrit Med J. 2011;17.

10. Sreeramareddy CT, Panduru KV, Verma SC, Joshi HS, Bates MN. Comparison of pulmonary and extrapulmonary tuberculosis in Nepal-a hospital-based retrospective study. BMC Infect Dis. 2008;8:8.

11. Gunal S, Yang Z, Agarwal M, Koroglu M, Arıcı ZK, Durmaz R. Demographic and microbial characteristics of extrapulmonary tuberculosis cases diagnosed in Malatya, Turkey, 2001-2007. BMC Publ Health. 2011;11:154.

12. Antony SJ, Harrell V, Christie JD, Adams HG, Rumley RL. Clinical differences between pulmonary and extrapulmonary tuberculosis: a 5-year retrospective study. $J$ Natl Med Assoc. 1995;87:187. 